\title{
Simulation on the Temperature Drop Rule of Hot Oil Pipeline
}

\author{
Enbin Liu ${ }^{*}$, , Liuting Yang ${ }^{1}$, Yong Jiang ${ }^{2}$, Ping Tang ${ }^{3}$, Jian Liu $^{4}$ and Yuhang Yang ${ }^{5}$ \\ ${ }^{1}$ Southwest Petroleum University, Chengdu, China \\ ${ }^{2}$ PetroChina Oil \& Gas Pipeline Control Center, Beijing, China \\ ${ }^{3}$ Sichuan Shida Energy Development Co., Ltd., Chengdu, China \\ ${ }^{4}$ Southwest Oil and Gas Field Branch, Chengdu, China \\ ${ }^{5}$ Offshore Oil Engineering Co., Ltd., Tianjin, China
}

\begin{abstract}
The heat transfer equation of oil, pipe wall and soil, combining with the boundary condition, connection condition and initial conditions to be the differential equations which can simulate the temperature drop rule of hot oil pipeline. Then the numerical solution method is used to solve, constructed difference equations. By the gauss elimination method, the temperature changing rule of hot oil pipeline after shutdown can be obtained, so we can determine the safety shutdown time and the restart pressure.
\end{abstract}

Keywords: Pipeline, temperature drop, model, simulation.

\section{INTRODUCTION}

Various accidents may occur inevitably during the operation process of the hot oil pipeline, such as natural disasters, power outages, planned maintenance and accident emergency repair to fault and so on, which contribute to pipeline shutdown. Due to the exothermic reaction of the oil in the pipeline after the shutdown, the temperature will gradually decline, viscosity will increase, the wax layer will thicken, and the liquidity of the oil will be affected. When the temperature of the save oil drop to a certain degree, it will be difficult to restart the pipeline, sometimes, condensing tube accidents may even happen [1-5].

Therefore, it is very important to do thermodynamic calculation after the shutdown of the hot oil pipeline, which helps us know the temperature drop situation and the temperature changing with time in various conditions after shutdown, and we can determine the safe shutdown time and the restarting pressure reasonably, which provides the scientific basis with safety production. It is very significant to the pipeline accident treatment, planned maintenance and the restart of the hot oil pipeline.

\section{THE THERMAL CALCULATION MODEL}

As shown in Fig. (1), it is the cross section diagram of a buried pipeline, assuming that there are $\mathrm{N}$ layers (wall, thermal insulation and protective layer, etc.) between the inside radius $R_{0}$ and the outside radius $R_{N}$, the inside radius of any layer is $R_{n-1}(n=1,2, \cdots, N)$.

*Address correspondence to this author at the Southwest Petroleum University, Chengdu, China; Tel: +86 - 28-83037039; Fax: +86 - 28-83032453; E-mail: Sunriselebpsb@163.com
The heat transfer problem of the oil can be described in polar coordinates $(r, \theta)$, and the heat transfer problem of semi-infinite soil can be described in Cartesian coordinate system ( xoy) [6,7].

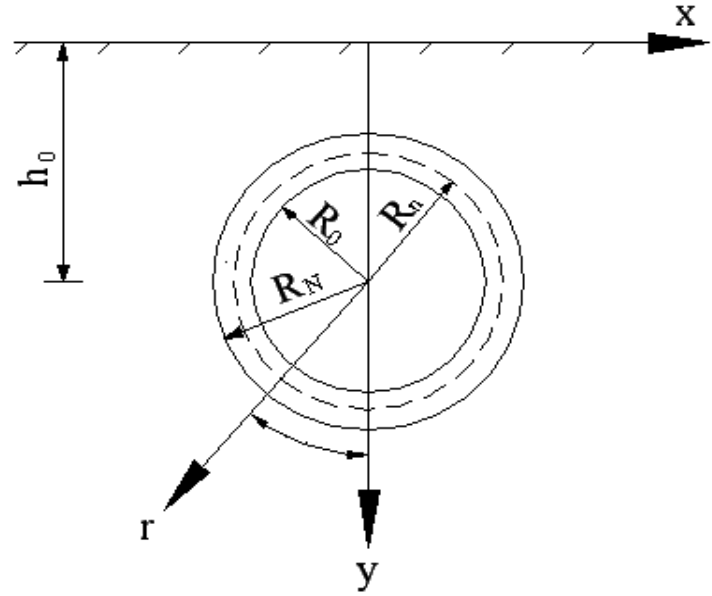

Fig. (1). The cross section diagram of a pipeline.

\section{The Heat Transfer Equation}

Based on the thermodynamic principle, the heat transfer equation of the hot oil pipeline is as follow:

$\rho c \frac{\partial T}{\partial t}=\frac{1}{r} \frac{\partial}{\partial r}\left(\lambda r \frac{\partial T}{\partial r}\right)+\frac{1}{r^{2}} \frac{\partial}{\partial \theta}\left(\lambda \frac{\partial T}{\partial \theta}\right)$

In which, $\rho$ is the oil density, $\mathrm{kg} / \mathrm{m}^{3} ; c$ is the oil heat capacity, $\mathrm{J} /\left(\mathrm{kg} \cdot{ }^{\circ} \mathrm{C}\right) ; T$ is the oil temperature, ${ }^{\circ} \mathrm{C} ; \lambda$ is the thermal conductivity of the oil, $\mathrm{W} /\left(\mathrm{m} \cdot{ }^{\circ} \mathrm{C}\right)$.

The heat transfer equation of the wall of the pipeline: 
$\rho_{n} c_{n} \frac{\partial T_{n}}{\partial t}=\frac{1}{r} \frac{\partial}{\partial r}\left(\lambda_{n} r \frac{\partial T_{n}}{\partial r}\right)+\frac{1}{r^{2}} \frac{\partial}{\partial \theta}\left(\lambda_{n} \frac{\partial T_{n}}{\partial \theta}\right)$

$(n=1,2, \cdots, N-1)$

In which, $\rho_{n}$ is the material density of the $\mathrm{n}$ layer of the pipeline, $\mathrm{kg} / \mathrm{m}^{3} ; c_{n}$ is the heat capacity of the $\mathrm{n}$ layer of the pipeline, $\mathrm{J} /\left(\mathrm{kg} \cdot{ }^{\circ} \mathrm{C}\right) ; T_{n}$ is the temperature of the $\mathrm{n}$ layer of the pipeline(wall, thermal insulation and protective layer, etc.), ${ }^{\circ} \mathrm{C} ; \lambda_{n}$ is the thermal conductivity of the $\mathrm{n}$ layer of the pipeline, $\mathrm{W} /\left(\mathrm{m} \cdot{ }^{\circ} \mathrm{C}\right)$.

The heat transfer equation of the soil is as follow:

$\rho_{g} c_{g} \frac{\partial T_{g}}{\partial t}=\frac{\partial}{\partial x}\left(\lambda_{g} \frac{\partial T_{g}}{\partial x}\right)+\frac{\partial}{\partial y}\left(\lambda_{g} \frac{\partial T_{g}}{\partial y}\right)$

In which, $\rho_{g}$ is the density of the soil, $\mathrm{kg} / \mathrm{m}^{3} ; c_{g}$ is the heat capacity of the soil, $\mathrm{J} /\left(\mathrm{kg} \cdot{ }^{\circ} \mathrm{C}\right) ; T_{g}$ is the temperature of the soil, ${ }^{\circ} \mathrm{C} ; \lambda_{g}$ is the thermal conductivity of the soil, $\mathrm{W} /\left(\mathrm{m} \cdot{ }^{\circ} \mathrm{C}\right)$.

\section{The Boundary Condition}

Considering that the maximum temperature of the oil in the cooling process of the pipeline will be misaligned (when $r=0, \frac{\partial T}{\partial r} \neq 0$ ), so:

$\lim _{r \rightarrow 0} r \frac{\partial T}{\partial r}=0$

The convection heat transfer equation between soil and outside air is as follow:

$\left.\frac{\partial T_{g}}{\partial y}\right|_{y=0}=-\frac{\alpha_{k}}{\lambda_{g}}\left(T_{g}-T_{0}\right)$

In which, $\alpha_{k}$ is the heat emission coefficient of the soil, $\mathrm{W} /\left(\mathrm{m}^{2} \cdot{ }^{\circ} \mathrm{C}\right) ; T_{0}$ is the temperature of the air, ${ }^{\circ} \mathrm{C}$.

\section{The Connection Condition}

Because the heat flux density of the oil is equal to the heat flux density at the innermost layer of the wall, a connection condition can be described as follow based on the convective heat transfer equation [7]:

$\left.\lambda \frac{\partial T}{\partial r}\right|_{r=R_{0}^{-}}=\left.\lambda_{1} \frac{\partial T_{1}}{\partial r}\right|_{r=R_{0}^{+}}=-\alpha_{Y}\left(T-T_{1}\right)$

In which, $\lambda_{1}$ is the thermal conductivity of the innermost layer of the pipe line wall, $\mathrm{W} /\left(\mathrm{m} \cdot{ }^{\circ} \mathrm{C}\right) . T_{1}$ is the temperature of the innermost layer, ${ }^{\circ} \mathrm{C} ; \quad \alpha_{Y}$ is the heat emission coefficient of the oil to the inner wall of pipeline, $\mathrm{W} /\left(\mathrm{m}^{2} \cdot{ }^{\circ} \mathrm{C}\right) ; R_{0}$ is The inner radius of the pipeline, $\mathrm{m}$.

The heat flux density of each layer of the pipeline is assumed to be equal, so: $\left.\lambda_{n} \frac{\partial T_{n}}{\partial r}\right|_{r=R_{n}^{-}}=\left.\lambda_{n+1} \frac{\partial T_{n+1}}{\partial r}\right|_{r=R_{n}^{+}}(n=1,2, \cdots, N-1)$

In which, $R_{n}$ is the outside radius of the pipeline, $\mathrm{m}$.

Because the boundary temperature of each layer of the pipeline is equal, so

$\left.T_{n}\right|_{r=R_{n}^{-}}=\left.T_{n+1}\right|_{r=R_{n}^{+}}(n=1,2, \cdots, N-1)$

Because the heat flux density of the outer layer of the oil pipeline is equal to the soil, so:

$\left.\lambda_{N} \frac{\partial T_{N}}{\partial r}\right|_{r=R_{N}^{-}}=\left.\lambda_{g} \frac{\partial T_{g}}{\partial r}\right|_{r=R_{N}^{+}}$

In which, $\lambda_{N}$ is The thermal conductivity of the outer layer of the oil pipeline, $\mathrm{W} /\left(\mathrm{m} \cdot{ }^{\circ} \mathrm{C}\right) ; T_{N}$ is The temperature of the outer layer of the pipeline, ${ }^{\circ} \mathrm{C} ; R_{N}$ is The radius of the outer layer of the pipeline, $\mathrm{m}$.

Because the heat flux density of the soil is equal to the heat flux density at the outer layer of the pipeline, so:

$\left.T_{N}\right|_{r=R_{N}^{-}}=\left.T_{g}\right|_{r=R_{N}^{+}}$

Initial Condition

$\left.T\right|_{t=0}=f(r, \theta)$

$\left.T_{n}\right|_{t=0}=f_{n}(r, \theta)$

$\left.T_{g}\right|_{t=0}=f_{g}(x, y)$

\section{NUMERIC SOLUTION OF THE MODEL}

Equation (1) is a heat transfer problem of the limited area described in the polar coordinates system. The oil in the pipeline is meshed as Fig. (2). The oil is divided into $J_{0}$ equal parts along the $\theta$ direction, $\Delta \theta=\frac{2 \pi}{J_{0}}$ and $\theta_{j}=j \Delta \theta\left(j=0,1, \cdots, J_{0}\right) ; \quad$ and $\quad M_{0} \quad$ equal parts along the $r$ direction, $\Delta r=\frac{R_{0}}{M_{0}+0.5}, r_{i}=(i+0.5) \Delta r$ $\left(i=0,1, \cdots, M_{0}\right)$.

The transfer equation of the oil, the wall and the soil will be saluted in the grid system shown in Fig. (2), forming one closed differential equations as follows:

$$
\begin{aligned}
& W_{1} T_{i, j}^{m+1}+W_{2} T_{i+1, j}^{m+1}+W_{3} T_{i-1, j}^{m+1}+W_{4} T_{i, j+1}^{m+1}+W_{5} T_{i, j-1}^{m+1}=\xi_{1} T_{i, j}^{m} \\
& \left(i=1,2, \cdots, M_{0}-1 ; j=1,2, \cdots, J_{0}-2\right) \\
& W_{1} T_{i, 0}^{m+1}+W_{2}^{\prime} T_{i+1,0}^{m+1}+W_{3}^{\prime} T_{i-1,0}^{m+1}+W_{4}^{\prime} T_{i, 1}^{m+1}+W_{5}^{\prime} T_{i, J_{0}-1}^{m+1}=\xi_{1}^{\prime} T_{i, 0}^{m} \\
& \left(i=1,2, \cdots, M_{0}-1\right)
\end{aligned}
$$




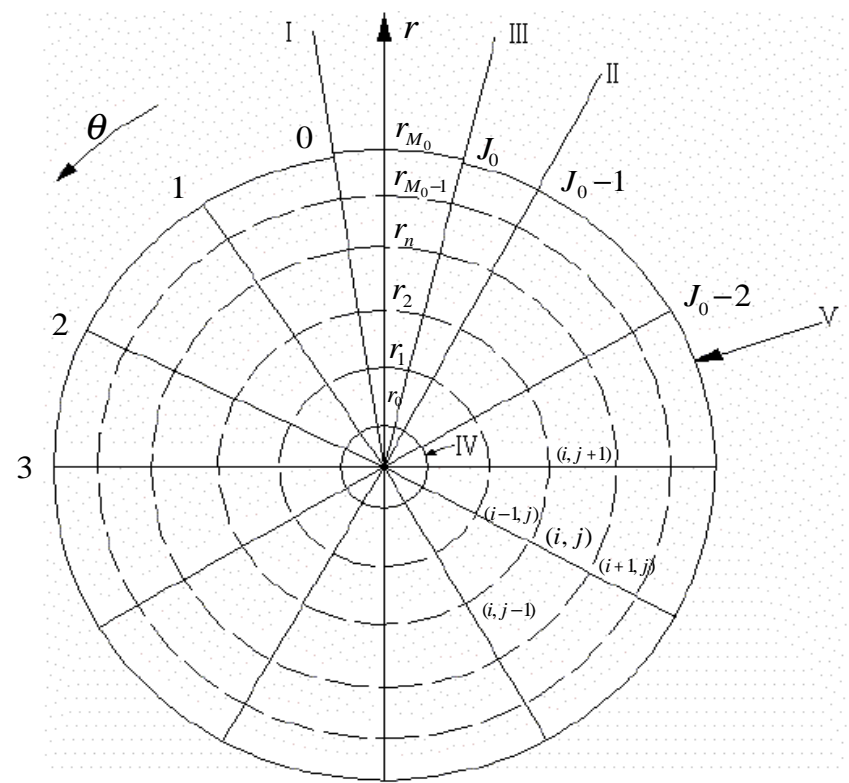

Fig. (2). The sketch map of the split-sectional pipeline.

$$
\begin{aligned}
& W_{1}^{\prime \prime} T_{i, J_{0}-1}^{m+1}+W_{2}^{\prime \prime} T_{i+1, J_{0}-1}^{m+1}+W_{3}^{\prime \prime} T_{i-1, J_{0}-1}^{m+1}+W_{4}^{\prime \prime} T_{i, 0}^{m+1}+W_{5}^{\prime \prime} T_{i, J_{0}-2}^{m+1}=\xi_{1}^{\prime \prime} T_{i, J_{0}-1}^{m} \\
& \left(i=1,2, \cdots, M_{0}-1\right) \\
& T_{i, J_{0}}^{m+1}-T_{i, 0}^{m+1}=0\left(i=0,1, \cdots, M_{0}\right) \\
& V_{1} T_{0, j}^{m+1}+V_{2} T_{1, j}^{m+1}+V_{3} T_{0, j+1}^{m+1}+V_{4} T_{0, j-1}^{m+1}=\mu_{1} T_{0, j}^{m} \\
& \left(j=1,2, \cdots, J_{0}-2\right) \\
& V_{1}^{\prime} T_{0,0}^{m+1}+V_{2}^{\prime} T_{1,0}^{m+1}+V_{3}^{\prime} T_{0,1}^{m+1}+V_{4}^{\prime} T_{0, J_{0}-1}^{m+1}=\mu_{1}^{\prime} T_{0,0}^{m} \\
& V_{1}^{\prime \prime} T_{0, J_{0}-1}^{m+1}+V_{2}^{\prime \prime} T_{1, J_{0}-1}^{m+1}+V_{3}^{\prime \prime} T_{0,0}^{m+1}+V_{4}^{\prime \prime} T_{0, J_{0}-2}^{m+1}=\mu_{1}^{\prime \prime} T_{0, J_{0}-1}^{m} \\
& j j(0,1) T_{M_{0}, j}^{m+1}-j j(0,1) T_{M_{0}-1, j}^{m+1}+j j(1,0) T_{0, j}^{m+1(1)} \\
& -j j(1,0) T_{1, j}^{m+1(1)}=0 \\
& \alpha_{M_{0}, j}^{m} T_{0, j}^{m+1(1)}-\left(\alpha_{M_{0}, j}^{m}+j j(0,1)\right) T_{M_{0}, j}^{m+1}+j j(0,1) T_{M_{0}-1, j}^{m+1}=0 \\
& T_{M}^{m+1}-\frac{1}{4}\left(T_{0,0}^{m+1}+T_{0, \frac{1}{4} J_{0}}^{m+1}+T_{0, \frac{1}{2} J_{0}}^{m+1}+T_{0, \frac{3}{4} J_{0}}^{m+1}\right)=0 \\
& W_{1}^{(n)} T_{i, j}^{m+1(n)}+W_{2}^{(n)} T_{i+1, j}^{m+1(n)}+W_{3}^{(n)} T_{i-1, j}^{m+1(n)}+W_{4}^{(n)} T_{i, j+1}^{m+1(n)} \\
& +W_{5}^{(n)} T_{i, j-1}^{m+1(n)}=\xi_{1}^{(n)} T_{i, j}^{m(n)} \\
& \left(n=1,2, \cdots, N-1 ; i=1,2, \cdots, M_{n}-1 ; j=1,2, \cdots, J_{0}-2\right) \\
& W_{1}^{\prime(n)} T_{i, 0}^{m+1(n)}+W_{2}^{\prime(n)} T_{i+1,0}^{m+1(n)}+W_{3}^{\prime(n)} T_{i-1,0}^{m+1(n)}+W_{4}^{\prime(n)} T_{i, 1}^{m+1(n)} \\
& +W_{5}^{\prime(n)} T_{i, J_{0}-1}^{m+1(n)}=\xi_{1}^{\prime(n)} T_{i, 0}^{m(n)} \\
& \left(n=1,2, \cdots, N-1 ; i=1,2, \cdots, M_{n}-1\right) \\
& W_{1}^{\prime \prime(n)} T_{i, J_{0}-1}^{m+1(n)}+W_{2}^{\prime \prime(n)} T_{i+1, J_{0}-1}^{m+1(n)}+W_{3}^{\prime \prime(n)} T_{i-1, J_{0}-1}^{m+1(n)}+W_{4}^{\prime \prime(n)} T_{i, 0}^{m+1(n)} \\
& +W_{5}^{\prime \prime(n)} T_{i, J_{0}-2}^{m+1}=\xi_{1}^{\prime \prime(n)} T_{i, J_{0}-1}^{m} \\
& \left(n=1,2, \cdots, N-1 ; i=1,2, \cdots, M_{n}-1\right)
\end{aligned}
$$

$$
\begin{aligned}
& T_{i, J_{0}}^{m+1(n)}-T_{i, 0}^{m+1(n)}=0 \quad\left(n=1,2, \cdots, N ; i=1,2, \cdots, M_{n}\right) \\
& j j(n, n+1) T_{M_{n}, j}^{m+1(n)}-j j(n, n+1) T_{M_{n}-1, j}^{m+1(n)} \\
& -j j(n+1, n) T_{1, j}^{m+1(n+1)}-j j(n+1, n) T_{0, j}^{m+1(n+1)}=0 \\
& \left(n=1,2, \cdots, N-1 ; j=1,2, \cdots, J_{0}\right) \\
& T_{M_{n}, j}^{m+1(n)}-T_{0, j}^{m+1(n+1)}=0 \\
& \left(n=1,2, \cdots, N-1 ; j=1,2, \cdots, J_{0}\right) \\
& e e 2 T_{M_{N}, j}^{m+1(N)}-e e 2 T_{M_{N}-1, j}^{m+1(N)}+e e 1 T_{g 0, j}^{m+1}-e e 1 T_{g 1, j}^{m+1}=0 \\
& \left(j=0,1, \cdots, J_{0}\right) \\
& T_{g 0, j}^{m+1}-T_{M_{N}, j}^{m+1(N)}=0\left(j=0,1, \cdots, J_{0}\right) \\
& K_{1} T_{g i, j}^{m+1}+K_{2} T_{g i+1, j}^{m+1}+K_{3} T_{g i-1, j}^{m+1}+K_{4} T_{g i, j+1}^{m+1}+K_{5} T_{g i, j-1}^{m+1}=K_{6} \\
& \left(i=1,2, \cdots, M_{\beta}-1 ; j=1,2, \cdots, J_{0}-2\right) \\
& K_{1}^{\prime} T_{g i, 0}^{m+1}+K_{2}^{\prime} T_{g i+1,0}^{m+1}+K_{3}^{\prime} T_{g i-1,0}^{m+1}+K_{4}^{\prime} T_{g i, 1}^{m+1}+K_{5}^{\prime} T_{g i, J_{0}-1}^{m+1}=K_{6}^{\prime} \\
& \left(i=1,2, \cdots, M_{\beta}-1\right) \\
& K_{1}^{\prime \prime} T_{g i, J_{0}-1}^{m+1}+K_{2}^{\prime \prime} T_{g i+1, J_{0}-1}^{m+1}+K_{3}^{\prime \prime} T_{g i-1, J_{0}-1}^{m+1}+K_{4}^{\prime \prime} T_{g i, 0}^{m+1} \\
& +K_{5}^{\prime \prime} T_{g i, J_{0}-2}^{m+1}=K_{6}^{\prime \prime} \\
& \left(i=1,2, \cdots, M_{\beta}-1\right) \\
& T_{g i, J_{0}}^{m+1}-T_{g i, 0}^{m+1}=0\left(i=0,1, \cdots, M_{\beta}-1\right) \\
& -(e 1+e 2) T_{g M_{\beta}, j}^{m+1}+e 1 T_{g M_{\beta}-1, j}^{m+1}=-e 2 T_{W}
\end{aligned}
$$

The unknown number of the equations is $\left(M_{0}+1\right)\left(J_{0}+1\right)+\sum_{n=1}^{N}\left(M_{n}+1\right)\left(J_{0}+1\right)+\left(M_{\beta}+1\right)\left(J_{0}+1\right)$,

which is equal to the number of the equations. And the equations are linear, which can be solved by Gauss Elimination Method.

Select the Gauss Elimination Method to solve differential equations. The differential equations showed before can be expressed in matrix form as follows:

$$
\left\{\begin{array}{l}
C_{11}^{(1)} T_{1}^{m+1}+C_{12}^{(1)} T_{2}^{m+1}+\cdots+C_{1 j}^{(1)} T_{j}^{m+1}+\cdots+C_{1 s z}^{(1)} T_{s z}^{m+1}=b_{1}^{(1)} \\
C_{21}^{(1)} T_{1}^{m+1}+C_{22}^{(1)} T_{2}^{m+1}+\cdots+C_{2 j}^{(1)} T_{j}^{m+1}+\cdots+C_{2 s z}^{(1)} T_{s z}^{m+1}=b_{2}^{(1)} \\
\cdots \cdots \cdots \cdots \cdots \cdots \cdots \cdots \cdots \cdots \cdots \cdots \cdots \cdots \cdots \\
C_{s z 1}^{(1)} T_{1}^{m+1}+C_{s z 2}^{(1)} T_{2}^{m+1}+\cdots+C_{s z j}^{(1)} T_{j}^{m+1}+\cdots+C_{s z s z}^{(1)} T_{s z}^{m+1}=b_{s z}^{(1)}
\end{array}\right.
$$

where, $C_{i j}^{(1)}$ is the temperature coefficient of point $j$ at the $t_{m+1}$ moment; $T_{j}^{m+1}$ is the temperature matrix at the $t_{m+1}$ moment; $b_{i}^{(1)}$ is the coefficient matrix related to the $t_{m}$ moment known before.

The formation of the matrix is: $C^{(1)} T^{m+1}=b^{(1)}$, with explanation that 


$$
C^{(1)}=\left[\begin{array}{cccc}
C_{11}^{(1)} & C_{12}^{(1)} & \cdots & C_{1 s z}^{(1)} \\
C_{21}^{(1)} & C_{22}^{(1)} & \cdots & C_{2 s z}^{(1)} \\
\cdots & \cdots & \cdots & \cdots \\
C_{s z 1}^{(1)} & C_{s z 2}^{(1)} & \cdots & C_{s z s z}^{(1)}
\end{array}\right], b^{(1)}=\left[\begin{array}{c}
b_{1}^{(1)} \\
b_{2}^{(1)} \\
\vdots \\
b_{s z}^{(1)}
\end{array}\right]
$$

besides $C^{(1)}$ is nonsingular phalanx.

Do the augmented matrix $\left[C^{(1)} \vdots b^{(1)}\right]$ elementary operation:

First, select principal component from the first row.

Premise: $\left|C_{i_{1}, 1}^{(1)}\right|=\max _{1 \leq i \leq s z}\left|C_{i 1}^{(1)}\right| \neq 0$, then exchange line $i_{1}$ and line 1 of $\left(C^{(1)}, b^{(1)}\right)$, and maintain the mark as $C_{i j}^{(1)}$ and $b_{i}^{(1)}$ respectively, then do the elimination.

If: $l_{i 1}=C_{i 1}^{(1)} / C_{11}^{(1)}(i=2,3, \cdots, s z), C_{i j}^{(2)}=C_{i j}^{(1)}-l_{i 1} C_{1 j}^{(1)}$ $(i, j=2,3, \cdots, s z), b_{i}^{(2)}=b_{i}^{(1)}-l_{i 1} b_{1}^{(1)}(i=2,3, \cdots, s z)$

Then $\left(A^{(1)}, b^{(1)}\right)$ equals:

$$
\left[\begin{array}{cccccc}
C_{11}^{(1)} & C_{12}^{(1)} & \cdots & C_{1 s z}^{(1)} & \vdots & b_{1}^{(1)} \\
0 & C_{22}^{(2)} & \cdots & C_{2 s z}^{(2)} & \vdots & b_{2}^{(2)} \\
\cdots & \cdots & \cdots & \cdots & \vdots & \cdots \\
0 & C_{s z 2}^{(2)} & \cdots & C_{s z s z}^{(2)} & \vdots & b_{s z}^{(2)}
\end{array}\right]=\left[C^{(2)}, b^{(2)}\right]
$$

Accordingly, the equation set can be expressed as $C^{(2)} T^{m+1}=b^{(2)}$.

Second, select a principle component from the second row of $C_{i 2}^{(2)}(i=2,3, \cdots, s z)$.

Make $\left|C_{i_{2}, 2}^{(2)}\right|=\max _{2 \leq i \leq s z}\left|C_{i 2}^{(2)}\right| \neq 0$, exchange line $i_{2}$ and line 2 of $\left(C^{(2)}, b^{(2)}\right)$, and maintain the mark as $C_{i j}^{(2)}, b_{i}^{(2)}$ respectively, then do the elimination.

If $\quad l_{i 2}=C_{i 2}^{(2)} / C_{22}^{(2)}(i=3,4, \cdots, s z), C_{i j}^{(3)}=C_{i j}^{(2)}-l_{i 2} C_{2 j}^{(2)}$ $(i, j=3,4, \cdots, s z), b_{i}^{(3)}=b_{i}^{(2)}-l_{i 2} b_{2}^{(2)}(i=3,4, \cdots, s z)$.

Then $\left(C^{(2)}, b^{(2)}\right)$ can be expressed as the following forms:

$$
\left[\begin{array}{ccccccc}
C_{11}^{(1)} & C_{12}^{(1)} & C_{13}^{(1)} & \cdots & C_{1 s z}^{(1)} & \vdots & b_{1}^{(1)} \\
0 & C_{22}^{(2)} & C_{23}^{(2)} & \cdots & C_{2 s z}^{(2)} & \vdots & b_{2}^{(2)} \\
0 & 0 & C_{33}^{(3)} & \cdots & C_{3 s z}^{(3)} & \vdots & b_{3}^{(3)} \\
\cdots & \cdots & \cdots & \cdots & \cdots & \cdots & \cdots \\
0 & 0 & C_{n 3}^{(3)} & \cdots & C_{s z s z}^{(3)} & \cdots & b_{s z}^{(3)}
\end{array}\right]=\left[C^{(3)}, b^{(3)}\right]
$$

Accordingly, the equation set can be expressed as $C^{(3)} T^{m+1}=b^{(3)}$.

Do as the steps below until $s z-1$, the results can be showed as:

$$
\left[\begin{array}{ccccccc}
C_{11}^{(1)} & C_{12}^{(1)} & C_{13}^{(1)} & \cdots & C_{1 s z}^{(1)} & \vdots & b_{1}^{(1)} \\
& C_{22}^{(2)} & C_{23}^{(2)} & \cdots & C_{2 s z}^{(2)} & \vdots & b_{2}^{(2)} \\
& & C_{33}^{(3)} & \cdots & C_{3 s z}^{(3)} & \vdots & b_{3}^{(3)} \\
& & & \ddots & \vdots & \vdots & \vdots \\
& & & & C_{s z s z}^{(s z)} & \vdots & b_{s z}^{(s z)}
\end{array}\right]=\left[C^{(s z)}, b^{(s z)}\right]
$$

Finally, the original equation set can be expressed as $C^{(s z)} T^{m+1}=b^{(s z)}$.

Namely, the step $\mathrm{k}(k=1,2, \cdots, s z-1)$ can be expressed as follows:

Select a principle component from the row k, $C_{i, k}^{(k)}(i=k+1, k+2, \cdots, s z)$.

Make $\left|C_{i_{k}, k}^{(k)}\right|=\max _{k \leq i \leq s z}\left|C_{i, k}^{(k)}\right| \neq 0$, exchange the line $i_{k}$ and line $\mathrm{k}$ of $\left(C^{(k)}, b^{(k)}\right)$, and maintain the mark as $C_{i j}^{(k)}, b_{i}^{(k)}$ respectively, then do the elimination.

Make $l_{i k}=C_{i k}^{(k)} / C_{k k}^{(k)}(i=k+1, k+2, \cdots, s z)$,

$C_{i j}^{(k+1)}=C_{i j}^{(k)}-l_{i k} C_{k j}^{(k)}(i, j=k+1, k+2, \cdots, s z)$,

$b_{i}^{(k+1)}=b_{i}^{(k)}-l_{i k} b_{k}^{(k)}(i=k+1, k+2, \cdots, s z)$.

After we get the equivalent equation sets $C^{(s z)} T^{m+1}=b^{(s z)}$, assume $C^{(1)}$ as nonsingular phalanx, then take it back to the equation step by step, finally we can get the solution of the original equation set, which is

$$
\left\{\begin{array}{l}
T_{s z}^{m+1}=b_{s z}^{(s z)} / C_{s z z z}^{(s z)} \\
T_{k}^{m+1}=\left(b_{k k}^{(k)}-\sum_{j=k+1}^{s z} C_{k j}^{(k)} T_{j}^{m+1} / C_{k k}^{(k)}(k=s z-1, s z-2, \cdots, 1)\right.
\end{array}\right.
$$

\section{APPLICATIONS}

For a buried hot oil pipeline, the diameter is $\Phi 355.6 \times 6.4$, the flow is $150 \times 10^{4} \sim 320 \times 10^{4} \mathrm{t} / \mathrm{a}$, and the Oil freezing point is $32^{\circ} \mathrm{C}$. The temperature of the soil near the pipeline is $15^{\circ} \mathrm{C}$ in summer, while $4^{\circ} \mathrm{C} \sim 5^{\circ} \mathrm{C}$ in winter and $8^{\circ} \mathrm{C} \sim 9^{\circ} \mathrm{Cin}$ spring, autumn respectively. The inlet oil temperature is $48 \sim 61{ }^{\circ} \mathrm{C}$, the outlet oil temperature is $72^{\circ} \mathrm{C}$. Figs. (3-5) are the temperature change of the pipeline over time after shutdown, which help us identify the safety shutdown time according to the nature of the oil properties.

We think the pipeline is safety when the lowest temperature of pipeline is $3{ }^{\circ} \mathrm{C}$ more than freezing point. The safety shutdown time is shown in Table $\mathbf{1}$, we can see that safety shutdown time is 22 hours in summer, while 15 hours in winter and 18 hours in spring, autumn respectively.

Table 1. Safety Shutdown Time

\begin{tabular}{|l|c|c|c|}
\hline & Winter & Spring and Autumn & Summer \\
\hline \hline Safety shutdown time, $\mathrm{h}$ & 15 & 18 & 22 \\
\hline
\end{tabular}




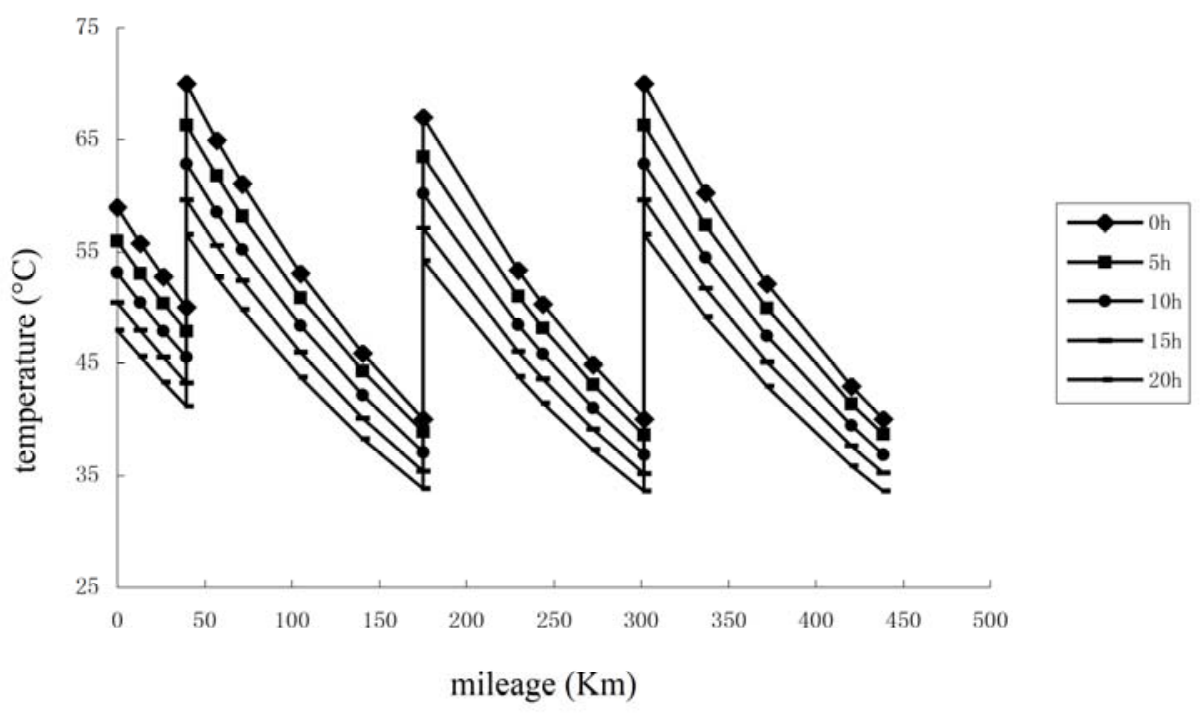

Fig. (3). The temperature drop diagram of the pipeline in spring and autumn.

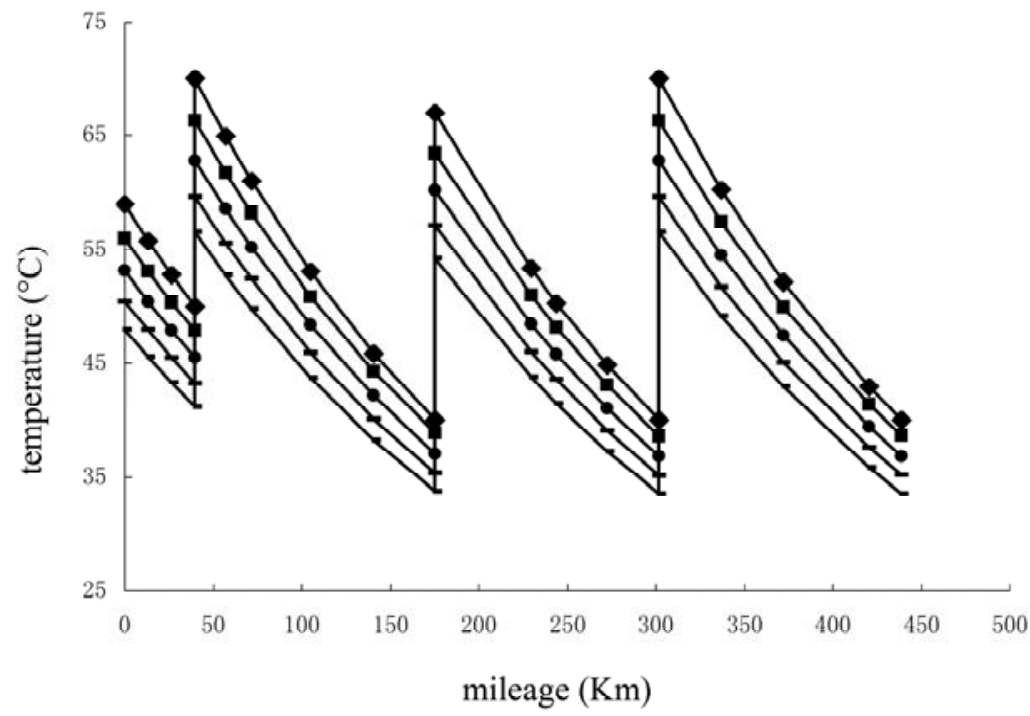

Fig. (4). The temperature drop diagram of the pipeline in summer.

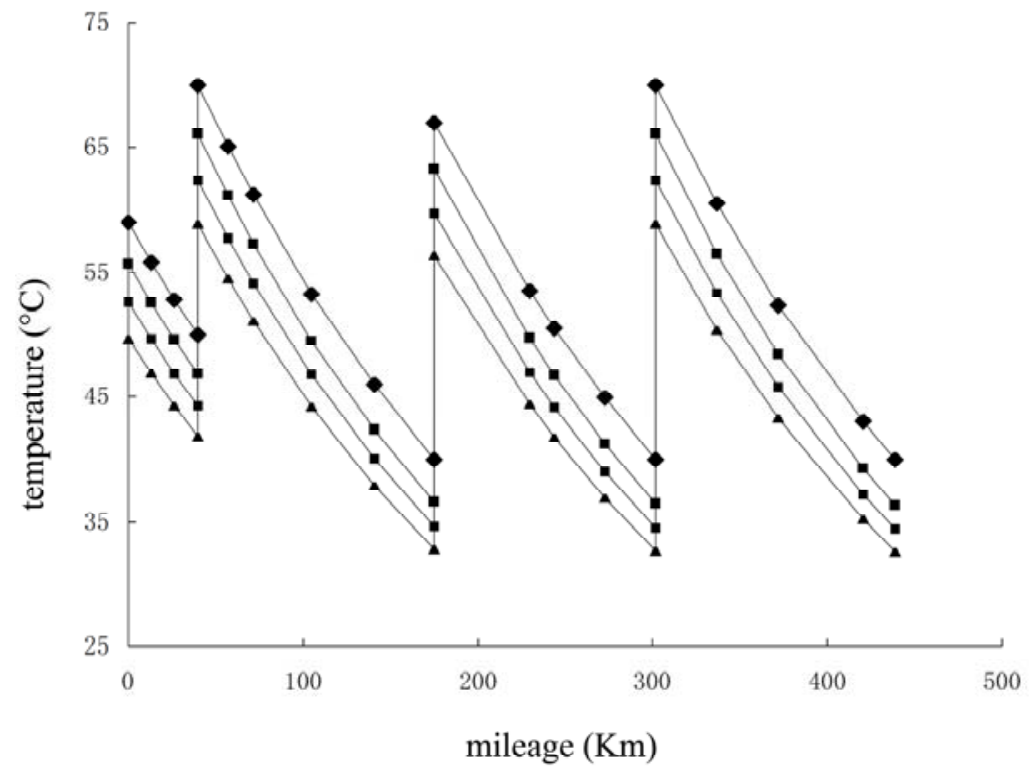

Fig. (5). The temperature drop diagram of the pipeline in winter. 


\section{CONCLUSION}

Combined with the boundary conditions, connected conditions and initial conditions, the heat transfer equations of oil in hot oil pipeline, pipe wall and soil are assembled to constitute differential equations for temperature drop analysis after hot oil pipeline shutdown. The above differential equations are solved with numerical method by constructing differential equations which can be solved with Gaussian elimination method. Thus, the temperature variation after the hot oil pipeline shutdown is obtained, providing the calculation basis for reasonably determining the safe shutdown time and the required re-starting pressure.

\section{CONFLICT OF INTEREST}

The authors confirm that this article content has no conflict of interest.

\section{ACKNOWLEDGEMENTS}

This paper is a project supported by the special fund of China's central government for the development of local colleges and universities_- the project of national firstlevel discipline in Oil and Gas Engineering, and sub-project of National science and technology major project of China (No.2011ZX05054).

\section{REFERENCES}

[1] Luo, T.H. Rheological properties of waxy crude oil and its pipeline transportation, Petroleum Industry Press, 1991.

[2] Bin, B.E. The highly viscous and pour point crude oil and products pipeline transportation. Petroleum Industry Press, 1997.

[3] Zhang, F. Pipelining blended crude oil by application of pour point depressant in Ma-Hue and Hong-Hue lines. Oil Gas. Storage Transport., 1988, 5, 21-24.

[4] Yang, X.H.; Zhang, G.Z. Pipeline design and management, Petroleum University Press, 1996.

[5] Huang, F.Q. The thermal calculation of underground pipeline. Petrol. Technol., 1980, 1, 77-91.

[6] Li, C.J. Numerical analysis of heated crude oil pipeline during shutdown. Oil Gas Storage Transport., 2001, 7, 28-31.

[7] Xie, Y.Y. The determination of safe shutdown time for waxy crude oil pipeline, Oil Gas Storage Transport., 2001, 8, 22-23. 